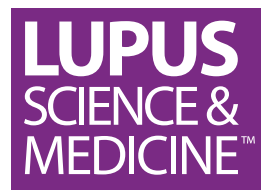

\title{
Illness representations of systemic lupus erythematosus and systemic sclerosis: a comparison of patients, their rheumatologists and their general practitioners
}

Seher Arat, ${ }^{1}$ Jan L Lenaerts, ${ }^{2,3,4}$ Ellen De Langhe, ${ }^{1,4}$ Patrick Verschueren, ${ }^{1,4}$ Philip Moons, ${ }^{5,6}$ Joris Vandenberghe, ${ }^{7,8}$ Veerle Taelman, ${ }^{4}$ Rene Westhovens ${ }^{1,4}$
To cite: Arat S, Lenaerts JL, De Langhe $\mathrm{E}$, et al. Illness representations of systemic lupus erythematosus and systemic sclerosis: a comparison of patients, their rheumatologists and their general practitioners. Lupus Science \& Medicine 2017;4:e000232. doi:10.1136/ lupus-2017-000232

Received 27 June 2017 Revised 22 August 2017 Accepted 12 0ctober 2017

CrossMark

For numbered affiliations see end of article.

Correspondence to Seher Arat; seher.arat@ kuleuven.be

\section{ABSTRACT}

Objective Discrepancies in illness representations between patients and physicians result in treatment difficulties, decreased well-being of patients and misunderstandings and disrupted communication. Hence, the objective of this study was to compare illness perceptions of individual patients with systemic lupus erythematosus (SLE) and systemic sclerosis (SSc), their rheumatologists and their general practitioners (GPs) and explore potential differences.

Methods This study has a cross-sectional design. Patients with SLE and SSc, who were followed at the rheumatology department of the University Hospitals Leuven (Belgium), completed the revised IIIness Perception Questionnaire which measures patients' perceptions of their condition and captures nine dimensions. Physicians completed the Revised IIIness Perception Questionnaire for Healthcare Professionals which consists of seven dimensions and measures perceptions of the healthcare professional regarding the disease of their patients. Intraclass correlation was performed to examine relationships between pairs of respondents; Cohen's d was used for estimating the magnitude of the difference.

Results Questionnaires were sent to 284 patients of whom 241 (113 SSc and 128 SLE patients) were included. Five rheumatologists and 160 GPs participated. For both diseases, positive correlations were found for 'consequences', 'illness coherence' and 'emotional representations' among patients, rheumatologists and GPs. GPs scored higher on the 'consequences' of these diseases for the patient ( $d=0.71$ for SLE; $d=0.80$ for SSc). Differences between rheumatologists and GPs were small for SSc and moderate to large for 'consequences' $(\mathrm{d}=0.56)$ and 'timeline acute/chronic' ( $\mathrm{d}=0.95)$ in SLE with higher scores for GPs.

Conclusions For both diseases and among the three groups, significant correlations are detected for the dimensions 'consequences', 'illness coherence' and 'emotional representations'. Differences between rheumatologists and GPs were mainly detected in the case of SLE patients. This can have implications for the collaboration between these two groups of physicians in daily clinical practice.
Clinical trial registration NCT02655640; Pre-results.

\section{INTRODUCTION}

Systemic lupus erythematosus (SLE) and systemic sclerosis (SSc) are severe and complex chronic autoimmune diseases characterised by multiple-organ involvement, a heterogeneous presentation and an unpredictable disease course often leading to important morbidity and mortality. ${ }^{12}$ Both diseases can have an important impact on patients' quality of life and the ability to carry out activities at home or at work due to pain, decreased physical functioning, fatigue and dyspnoea. $^{3-6}$ These progressive or recurrent symptoms might influence the perceptions patients have about their condition.

Ideas about illness are an essential part of the self-regulation model, which proposes that behaviour in relation to illness depends on an individual's perception or representation of his/her condition. In this model, Leventhal and colleagues ${ }^{7}$ postulated that illness representations consist of five elements: identity (symptoms), cause, consequences (effects on life), timeline (duration) and controllability or cure of the condition. Studies in patients with SLE have demonstrated that illness perceptions are related to outcomes such as changes in psychological well-being over time, ${ }^{8}$ sexual (dys)functioning, ${ }^{9}$ (non-) adherence to therapy ${ }^{10}$ and in those with $\mathrm{SSc}$ to physical and mental health but not to disease-related characteristics. ${ }^{11} 12$

Most studies only focus on patients' illness perceptions and their association with clinical or patient-related outcomes, ${ }^{13}$ but the extent to which healthcare providers' ideas about the consequences of a chronic disease in 
specific patients match with those expressed by individual patients is unknown. This could be important because the management of SLE and SSc requires a therapeutic relationship between patients and providers over years, which makes adequate healthcare a joint responsibility of both providers and patients. Moreover, it is possible that because of this relationship for years, physicians' perceptions can be influenced by patients and vice versa.

In one of the first studies ${ }^{14}$ describing beliefs about arthritis in patients and physicians (most of them were rheumatologists), differences were detected about what physicians think patients believe and what patients actually believe about causes of arthritis or what helps in arthritis. In a study about epilepsy and seizure disorders, there were differences between the illness perceptions of patients and their doctors, especially about the controllability of the condition, which could represent barriers to successful clinical management. ${ }^{15}$ In another study about breast cancer, ${ }^{16}$ medical professionals' perceptions of the consequences of treatment and duration of cancer did not match patients' beliefs: oncology nurses underestimated, whereas radiation therapists overestimated the impact of treatment and perceived duration of the disease. A study in osteoarthritis and diabetes showed that incongruence in patients and general practitioners' (GPs') perceptions regarding stressors accompanying chronic disease is larger in diseases with a less clear treatment policy and may influence healthcare use and physical and mental functioning. ${ }^{17}$

So, detecting discrepancies between healthcare providers and patients is of utmost importance because these may lead to problems in treatment, decreased wellbeing of patients ${ }^{18}$ and misunderstandings and disrupted communication. ${ }^{171920}$ Differences in illness perceptions among patients with systemic autoimmune diseases, rheumatologists and GPs are likely to be relevant because the knowledge and disease-related experience of these three groups is different. This is the first study to investigate these differences and to attempt a direct comparison between physicians and particular patients with SLE and SSc they care for. Hence, the aim of this study was to investigate similarities and differences in illness perceptions of individual patients with SLE and SSc with that of their rheumatologists and GPs.

\section{METHODS \\ Design}

The present study has a cross-sectional design. However, it was part of a larger longitudinal project in patients with SLE and SSc. This study has been registered in clinicaltrials.gov with ID number NCT02655640. The data for the current evaluation were those collected at baseline between November 2015 and February 2016.

\section{Study population}

The study population consisted of patients, rheumatologists and GPs. A total of 284 patients with SLE and SSc, who were in follow-up at the University Hospitals Leuven (Belgium) and fulfilled the inclusion criteria, were approached and invited for participation. The inclusion criteria were as follows: the patient's medical and cognitive condition allows him/her to complete questionnaires; the patient has no severe psychiatric problems; the patient is proficient in Dutch and able to complete the questionnaires in Dutch. The five rheumatologists who were asked for participation worked at the systemic diseases care programme at the rheumatology department of the University Hospitals Leuven. In addition to the rheumatologists, the patients' GPs were also asked to participate.

\section{Procedure}

Patients received a letter with information about the goal of the study, a questionnaire pack and an informed consent form together with a prestamped envelope. Patients were asked to complete the informed consent and the questionnaire pack and return it within 2 weeks.

After the patients gave informed consent, the treating rheumatologist and GP of each patient were approached. The physicians were asked to fill out an illness perception questionnaire developed for healthcare professionals after completing an informed consent form. While completing the questionnaire, they had to rely on the most recent medical and psychosocial situation of the patient. The physicians were requested to complete the questionnaires as soon as possible after the patient consulted them. A maximum interval of 6 months between the consultation of the patient and completion of the questionnaire by the physician was allowed. Additionally, for both patients and physicians, three reminders were sent after 3, 5 and 7 weeks. After 9 weeks, patients were contacted by telephone if they were persistent non-responders.

\section{Measures}

\section{Demographic characteristics}

Age, gender, educational level, employment status, social status and living situation were collected from SLE and SSc patients (see table 1).

\section{Clinical data}

Disease duration and disease activity were measured. In SLE patients, disease activity was assessed by the Systemic Lupus Erythematosus Disease Activity Index (SLEDAI) score with the Safety of Estrogens in Lupus Erythematosus National Assessment (SELENA) modification (ie, SELENA-SLEDAI) ${ }^{21}$ The SLEDAI is a valid and reliable index, which measures disease activity within the last 10 days $^{22}$ including 24 weighted objective clinical and laboratory variables. Disease activity can range from 0 to 105. The following activity categories have been defined on the basis of SLEDAI scores: no activity (SLEDAI 0), mild activity (SLEDAI 1-5), moderate activity (SLEDAI 6-10), high activity (SLEDAI 11-19) and very high activity $($ SLEDAI $\geq 20) .{ }^{23}$ 
Table 1 Demographic and clinical characteristics of patients

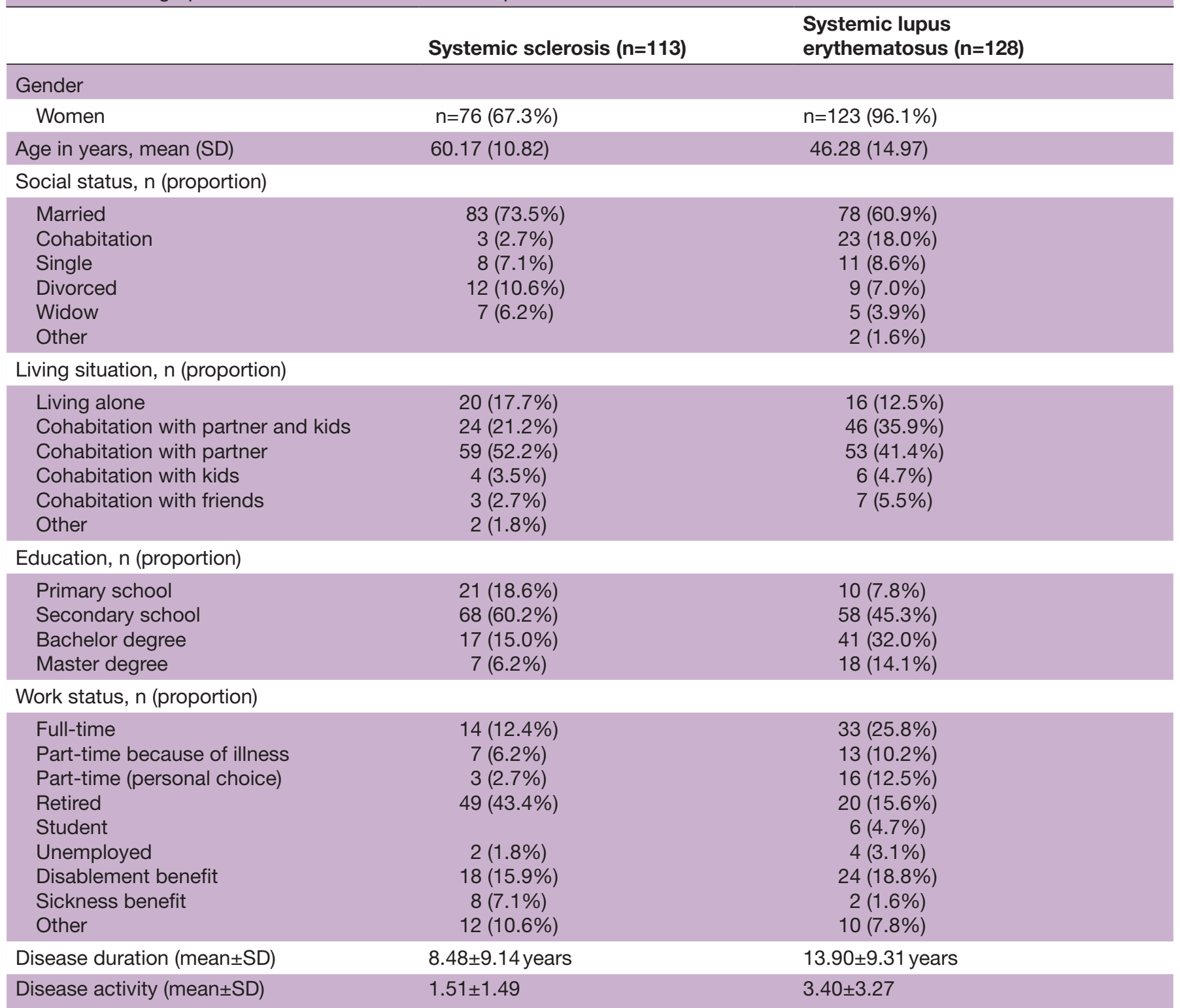

In SSc patients, the SSc disease activity index $(2003)^{24}$ was used for measuring disease activity. This is a preliminary validated index to assess disease activity in SSc which consists of clinical and laboratory measures of disease activity as well as measures of disease activity being assessed by the patient only. It captures 10 weighted measures, and the scores have a range from 0 to 10 . SSc is considered to be active if the disease activity is $\geq 3$.

IIIness perceptions of patients and physicians

Patients completed the Dutch version ${ }^{25}$ of the revised Illness Perception Questionnaire (IPQ-R) developed by Moss-Morris and colleagues. ${ }^{26}$ This questionnaire measures perceptions of patients regarding their disease and consists of nine dimensions or subscales: an illness identity dimension, seven illness perception subscales and a causal attributions dimension. It has demonstrated good reliability and validity across several illness groups and is one of the most widely applied instruments for assessing perceptions about illness. ${ }^{27}$

For the purpose of this study, we only used the seven illness perception subscales (38 items) that include views about how long the disease will last (timeline acute/ chronic), the recurrent nature of the condition (timeline cyclical), perceived consequences of the condition, perceptions of personal control and treatment control, patient's overall illness comprehension (illness coherence) and emotional representations. The items for all subscales are rated by the patient on a 5-point Likert scale from 1 ('strongly disagree') to 5 ('strongly agree'). Scores were calculated as the sum of the items per scale (as in the original publication).

The physicians completed the Revised Illness Perception Questionnaire for Healthcare Professionals (IPQ-R HP).${ }^{28}$ The IPQ-R HP ${ }^{28}$ is an adapted version of the IPQ-R ${ }^{26}$ 
which is devised to be completed by healthcare professionals. It is a 38-item questionnaire rated on a 5-point Likert scale from 1 ('strongly disagree') to 5 ('strongly agree') and consists of the same seven dimensions as described above. The key characteristic of the IPQ-R HP is that healthcare professionals are asked to indicate what they think what the perceptions of the particular patient are. Sample items read as: 'The illness of my patient has major consequences on his/her life'; 'The illness of my patient will last for a long time'; or 'The symptoms of the condition of my patient are puzzling to him/her'.

\section{Statistical analysis}

Sociodemographic variables, clinical variables and self-reported data from the questionnaires were summarised using descriptive statistics such as frequencies, means and SDs. To check the normality of the data, the Kolmogorov-Smirnov test was used.

Intraclass correlation was performed to examine agreement among the three groups of respondents regarding the seven illness perception dimensions: rheumatologists and their patients, GPs and their patients, and rheumatologists and GPs. Cut-off values for the intraclass correlation are trivial $<0.1$, small $0.1-0.29$, moderate $0.3-0.49$, large $0.5-0.69$, very large $0.7-0.89$, almost perfect $>0.9 .^{29}$

A paired t-test was conducted to detect differences in these seven dimensions among the three pairs of respondents as described above. The choice for a paired t-test was made on the basis of the distribution of the data and the link between patients and physicians and rheumatologists and GPs.

In addition, to appraise the magnitude of potential differences in perceptions between the groups, Cohen's $\mathrm{d}$ was calculated to estimate how large the difference was between the mean scores on the dimensions for each group. The cut-off values for Cohen's $d$ are as follows: small $0.20-0.50$, medium $0.50-0.80$ and large $\geq 0.80 .{ }^{30}$

Analyses were carried out with SPSS V.24.0 (SPSS, Chicago, Illinois, USA).

\section{Ethical approval}

Ethical approval was obtained from the institutional review board of the University Hospitals Leuven (ID no. B322201526067).

\section{RESULTS}

\section{Respondents}

Questionnaires were sent to 284 patients who fulfilled the inclusion criteria, 241 of which participated (113 SSc patients and 128 SLE patients). The five rheumatologists and GPs of these patients were also asked to participateasked for participation. The rheumatologists completed together a total of 229 questionnaires. A total of 240 GPs received questionnaires, 160 of which returned completed questionnaires (response rate 66.7\%) (see figure 1 for detailed information). The demographic and clinical information of the patients is shown in table 1 .

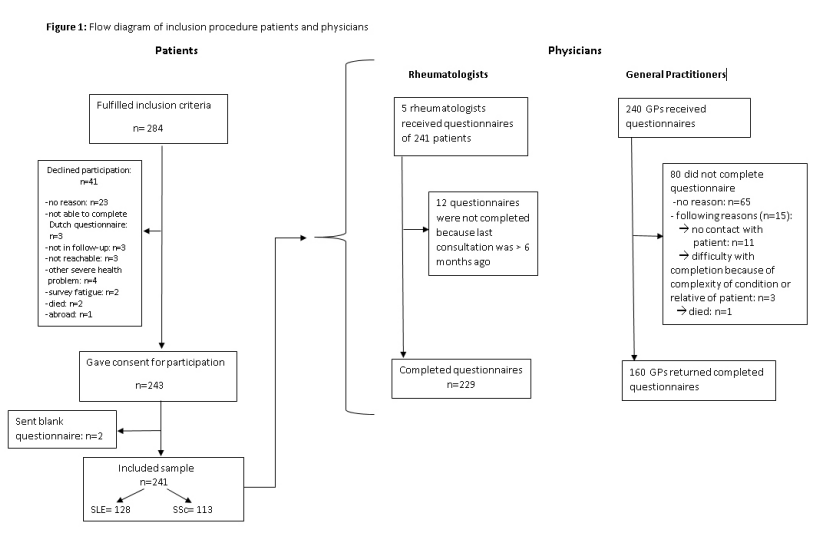

Figure 1 Flow diagram of inclusion procedure of patients and physicians.

The group of the rheumatologists consisted of two women and three men. The group of the GPs consisted of 99 men and 61 women. The gender distribution in the group of the non-responders was comparable to that of the responders $(60.0 \%$ vs $61.9 \%)$. No information on other characteristics was available.

\section{Comparisons among illness representations of SLE patients, their rheumatologists and their GPS}

Between patients and rheumatologists, a large positive intraclass correlation was found for the perceived consequences of SLE. Moderate intraclass correlations were observed for controllability by treatment, illness coherence and the emotional impact of SLE (see table 2). Looking at the Cohen's d, differences between patients and their rheumatologists were futile or small for all dimensions, except for timeline cyclical, on which the patients scored higher with a moderate difference.

Between patients and GPs, moderate positive correlations were found for the perceived consequences, illness coherence and the emotional impact of SLE. A moderately large difference was found for consequences, on which GPs scored higher than their patients.

Between rheumatologists and GPs, moderate but statistically significant correlations were found for the perceived consequences, chronicity of the time course, illness coherence and the emotional impact of SLE. For consequences, the difference in mean score was moderate, with higher scores in GPs than in rheumatologists, and for timeline acute/chronic, the difference was large.

Comparisons between illness representations of SSc patients, their rheumatologists and their GPS

Also for SSc, moderate positive intraclass correlations were found between patients and rheumatologists on the consequences dimension, illness coherence and emotional representations (see table 3). Patients scored lower in comparison with rheumatologists, with a moderate difference for the consequences dimension. 

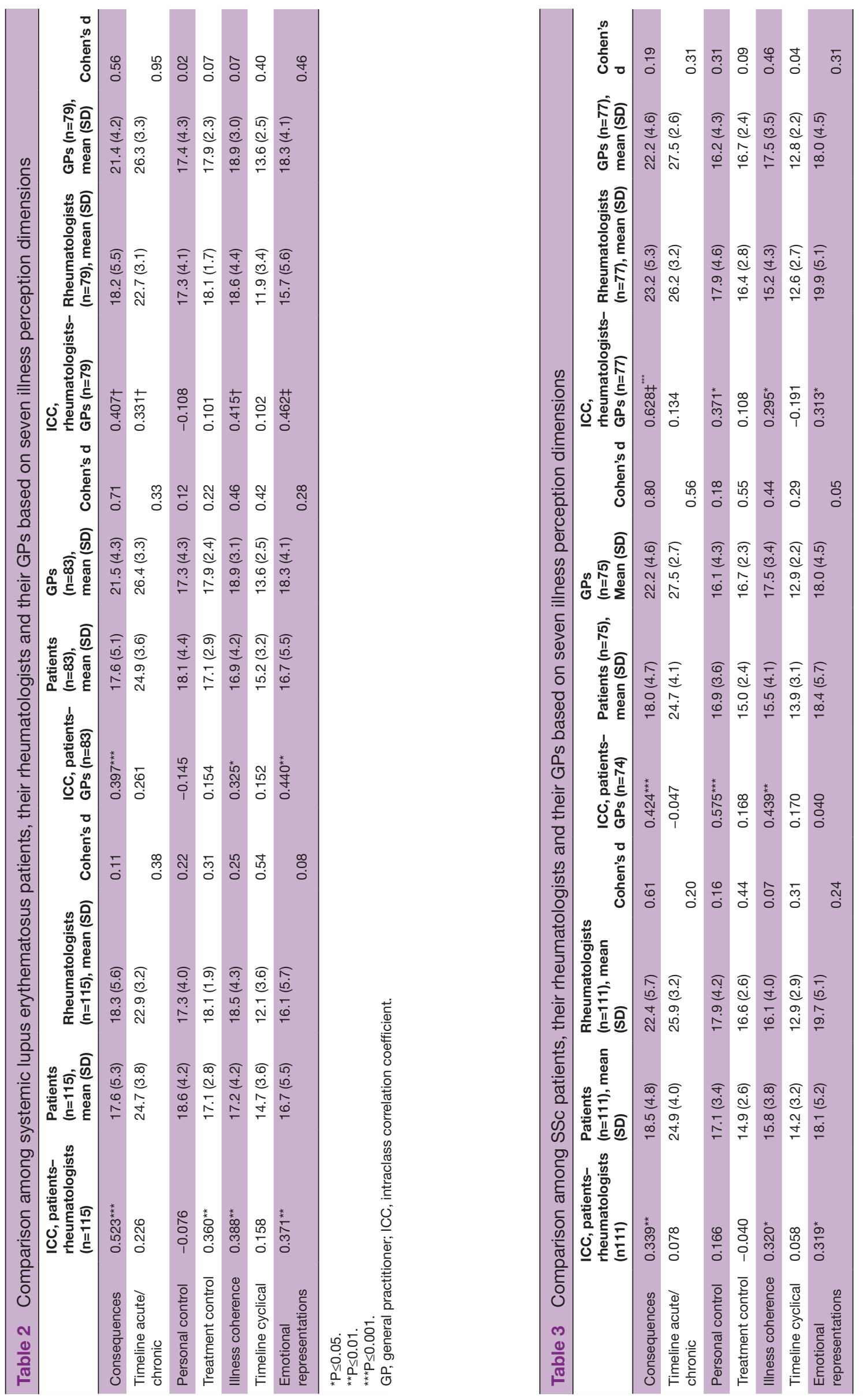
Between patients and GPs, moderate positive correlations were found for consequences and illness coherence and large intraclass correlations for personal control. Patients had lower mean scores on the perceived consequences with a large difference for consequences and moderate difference for timeline acute/chronic and illness coherence.

Between rheumatologists and GPs, large positive correlations were found for the perceived consequences; and moderate correlations, for personal control, illness coherence and the emotional impact of SSc. A large difference was found for consequences, on which GPs scored higher than patients. For timeline acute/chronic and treatment control, a moderate difference was found with also higher scores for GPs than patients. The detected differences between rheumatologists and GPs were small for all dimensions.

\section{DISCUSSION}

The aim of this paper was to explore commonalities and differences in perceptions among patients with SLE and SSc, their rheumatologists and their GPs on the individual representations of their illness.

For both diseases, we found moderate to large correlations in the consequences, illness coherence and emotional representations dimension among patients, rheumatologists and GPs. The GPs scored higher on these dimensions in comparison with the patients, but the difference was mostly small to moderate. They seemed to overestimate the consequences of these diseases for the patient, the understanding of these diseases by the patient and the emotional impact for SLE patients but not for SSc patients. Our results are in concordance with a number of studies in epilepsy ${ }^{15} 3132$ in which the neurologists score higher on the aforementioned dimensions than the patients. The rheumatologists scored also higher on these dimensions than the patients, but the differences were mostly small. Except in SLE, patients had the perception of a more recurrent time course than rheumatologists. This difference was moderate.

A closer look at comparisons between rheumatologists and GPs shows that, for SLE, GPs have higher scores on consequences, illness coherence, timeline acute/chronic and emotional representations and, for SSc, rheumatologists score higher than the GPs on these dimensions (except for illness coherence). This reflects that GPs consider SLE to be a much more severe condition than rheumatologists and patients do. This could be attributed to lack of knowledge or the fact that they rely on general information about SLE-for instance, coming from textbooks, which tend to put too much emphasis on severe SLE manifestations-when scoring the questionnaire.

For SSc, moderate to large correlations were found for personal control in both patients and GPs and GPs and rheumatologists. The difference between these groups was statistically small but can have clinical implications. The fact that there is a difference can be related to the rarity of SSc and interpatient differences, both in the type of controllable symptoms and the level of control, ${ }^{33}$ and that personal control is a construct of illness perceptions which is the most complex and multifactorial in relation to the other dimensions. ${ }^{34}$ Another explanation is that GPs might have lack of knowledge or limited patient contact and therefore rely more on the information provided by the patient. This emphasises the importance of the collaboration between GPs and rheumatologists for receiving and providing up-to-date disease-related information. In daily clinical practice, perceived control is based on knowledge about the disease-for instance, provided by a healthcare professional-and also on the patients' personal experience. This dimension is important because of several reasons. The first reason is that personal control is one of the dimensions that predicts outcomes and is easily changeable with interventions. Much more than the other dimensions, personal control can be altered by patient education sessions and self-management programs. ${ }^{35-37}$ Dedicated educational programs with an emphasis on the perceptions of patients, initiated by physicians, could reduce the knowledge deficit in the patients. Also, as the GP often remains the primary caregiver, it is crucial that the illness perceptions of both GP and rheumatologists are aligned. For this, communication modalities between these two groups of health professionals should be optimised.

The strengths of this study are that we had dyads of patients, rheumatologists and GPs and a high response rate. The number of GPs that participated was considerable. The study setting was a large university hospital in Belgium. The results of this study should be interpreted in light of some methodological limitations. A small number of GPs sent us a blank questionnaire with a note that they did not want to complete the IPQ-R HP because the patient visited them too long ago or not frequently so that they did not feel comfortable to judge in detail the patient's health situation. Another limitation is that the rheumatologists who completed the IPQ-R HP mentioned that completing the questionnaire was not always easy. Some dimensions such as timeline acute/ chronic, treatment control and personal control were mainly based on medical knowledge. Other dimensions such as the emotional representations and illness coherence were completed much more subjectively by putting themselves in place of the patient. So, the set-up of the IPQ-R HP needs further exploration.

Another point for consideration is the external validity of the study results. This study has been conducted in a large single centre which implies that the generalisability can be limited. Furthermore, the current study has a cross-sectional design which implies that it cannot establish the directionality of the associations between rheumatologists or GPs and patients. It is possible that the physicians' perception is influenced by the way a patient perceives and reports his condition or vice versa. Other implications for further 
research are that the uncovered differences in illness perceptions between patients and medical staff can now be studied for their impact on outcomes. The current study provides insights in illness perception dimensions that are important for inclusion in future research about the influence of illness perceptions on outcomes such as patient satisfaction, adherence, healthcare utilisation and others.

In conclusion, we can state that for both diseases and among patients, rheumatologists and GPs, significant correlations in illness perceptions are detected for the dimensions consequences, illness coherence and emotional representations. For SLE, we found that GPs perceived the consequences of SLE as more severe than patients and rheumatologists do and that GPs also perceived SLE as more chronic than rheumatologists. GPs perceived SSc as more severe, chronic and controllable by treatment than patients. These differences can have implications for the communication and collaboration in daily clinical practice.

\section{Author affiliations}

${ }^{1}$ Department of Development and Regeneration, Katholieke Universiteit Leuven, Skeletal Biology and Engineering Research Center, Leuven, Belgium ${ }^{2}$ Department of Rheumatology, Jessa Hospitals Hasselt, Hasselt, Belgium ${ }^{3}$ Department of Rheumatology, Reuma-Instituut Hasselt, Hasselt, Belgium ${ }^{4}$ Department of of Rheumatology, University Hospitals Leuven, Leuven, Belgium ${ }^{5}$ Department of Public Health and Primary Care, KU Leuven, Goteborg, Sweden ${ }^{6}$ Instituteof Health and Care Sciences, University of Gothenburg, Leuven, Belgium ${ }^{7}$ Department of Neurosciences, Katholieke Universiteit Leuven, Leuven, Belgium ${ }^{8}$ Department of of Psychiatry, University Hospitals Leuven, Leuven, Belgium

Acknowledgements We are very grateful to all general practitioners and patients who participated in this study.

Contributors SA, PM, JV and RW conceived the study and participated in its design and data analysis, interpretation of the findings, report writing, manuscript preparation and critically revising of the manuscript. JL, EDL, PV and VT were involved in the data collection, data analysis, interpretation of the findings and critically revising of the manuscript. All authors read and approved the final version of the manuscript.

Funding This research project was supported by the Belgian 'Fonds voor Wetenschappelijk Reuma Onderzoek/Fonds pour la Recherche Scientifique en Rhumatologie'.

\section{Competing interests None declared.}

Ethics approval Institutional Review Board of the University Hospitals Leuven. Provenance and peer review Not commissioned; externally peer reviewed. Data sharing statement № additional data are available.

Open Access This is an Open Access article distributed in accordance with the Creative Commons Attribution Non Commercial (CC BY-NC 4.0) license, which permits others to distribute, remix, adapt, build upon this work non-commercially, and license their derivative works on different terms, provided the original work is properly cited and the use is non-commercial. See: http://creativecommons.org/ licenses/by-nc/4.0/

(c) Article author(s) (or their employer(s) unless otherwise stated in the text of the article) 2017. All rights reserved. No commercial use is permitted unless otherwise expressly granted.

\section{REFERENCES}

1. Desbois AC, Cacoub P. Systemic sclerosis: an update in 2016. Autoimmun Rev 2016;15:417-26.

2. D'Cruz DP, Khamashta MA, Hughes GR. Systemic lupus erythematosus. Lancet 2007;369:587-96.
3. Schmeding A, Schneider M. Fatigue, health-related quality of life and other patient-reported outcomes in systemic lupus erythematosus. Best Pract Res Clin Rheumatol 2013;27:363-75.

4. Malcus Johnsson P, Sandqvist G, Bengtsson A, et al. Hand function and performance of daily activities in systemic lupus erythematosus. Arthritis Rheum 2008;59:1432-8.

5. Poole JL, Brandenstein J. Difficulty with daily activities involving the lower extremities in people with systemic sclerosis. Clin Rheumatol 2016;35:483-8.

6. Poole JL, Chandrasekaran A, Hildebrand K, et al. Participation in life situations by persons with systemic sclerosis. Disabil Rehabil 2015;37:842-5.

7 Leventhal H, Nerenz DR, Steele DJ. Illness representations and coping with health threats. In: Baum A, Taylor S, eds. Handbook of Psychology and Health. Volume IV: social psychological aspects of health, 1984:219-52.

8. Shortall E, Isenberg D, Newman SP. Factors associated with mood and mood disorders in SLE. Lupus 1995;4:272-9.

9. Daleboudt GM, Broadbent E, McQueen F, et al. The impact of illness perceptions on sexual functioning in patients with systemic lupus erythematosus. J Psychosom Res 2013;74:260-4.

10. Daleboudt GM, Broadbent E, McQueen F, et al. Intentional and unintentional treatment nonadherence in patients with systemic lupus erythematosus. Arthritis Care Res 2011;63:342-50.

11. Arat $\mathrm{S}$, Verschueren $\mathrm{P}$, De Langhe $\mathrm{E}$, et al. The association of illness perceptions with physical and mental health in systemic sclerosis patients: an exploratory study. Musculoskeletal Care 2012;10:18-28.

12. Richards HL, Herrick AL, Griffin K, et al. Systemic sclerosis: patients' perceptions of their condition. Arthritis Rheum 2003;49:689-96.

13. Hagger MS, Orbell S. A meta-analytic review of the common-sense model of illness representations. Psychol Health 2003;18:141-84.

14 Lorig KR, Cox T, Cuevas Y, et al. Converging and diverging beliefs about arthritis: Caucasian patients, Spanish speaking patients, and physicians. J Rheumatol 1984;11:76-9.

15. Whitehead K, Kandler R, Reuber M. Patients' and neurologists' perception of epilepsy and psychogenic nonepileptic seizures. Epilepsia 2013;54:708-17.

16 Buick DL, Petrie KJ, Weinman JA. Illness representations and breast cancer: Coping with radiation and chemotherapy. In: Petrie $\mathrm{K}$, Weinman J, eds. Perceptions of health and illness: Current research and applications. East Sussex: Routledge Taylor \& Francis, 1997:379-409.

17. Heijmans M, Foets M, Rijken M, et al. Stress in chronic disease: do the perceptions of patients and their general practitioners match? $\mathrm{Br}$ $J$ Health Psychol 2001;6:229-42.

18. Kleinman A. Culture, Illness, and Care. Ann Intern Med 1978;88:251.

19. Lunde IM. Patients' perceptions - a shift in medical perspective. Scand J Prim Health Care 1993:11:98-104.

20. van-der Hofstadt C, Rodríguez-Marín J, Quiles M, et al. Illness representation of arterial hypertension in a sample of health professionals and the general public. Psychol Health Med 2003;8:81-7.

21. Petri M, Kim MY, Kalunian KC, et al. Combined oral contraceptives in women with systemic lupus erythematosus. N Engl J Med 2005;353:2550-8.

22 Bombardier C, Gladman DD, Urowitz MB, et al. Derivation of the SLEDAI. A disease activity index for lupus patients. The Committee on Prognosis Studies in SLE. Arthritis Rheum 1992;35:630-40.

23 Cook RJ, Gladman DD, Pericak D, et al. Prediction of short term mortality in systemic lupus erythematosus with time dependent measures of disease activity. $J$ Rheumatol 2000;27:1892-5.

24. Valentini G, Bencivelli W, Bombardieri S, et al. European scleroderma study group to define disease activity criteria for systemic sclerosis. III. Assessment of the construct validity of the preliminary activity criteria. Ann Rheum Dis 2003;62:901-3.

25 Heijmans M, Rijken M, 2003. Validation of the IPQ-R in a large, representative Dutch sampleln:. Book of abstracts of the 17th Conference of the European Health Psychology Society. European health psychology. Gender, culture and health, Kos, Greece;24-27 Sep 2003; 14

26. Moss-Morris R, Weinman J, Petrie K, et al. The revised illness perception questionnaire (IPQ-R). Psychol Health 2002;17:1-16.

27. Maas $\mathrm{M}$, Taal $\mathrm{E}$, van der Linden $\mathrm{S}$, et al. A review of instruments to assess illness representations in patients with rheumatic diseases. Ann Rheum Dis 2009;68:305-9.

28 Arat S, Van den Zegel A, Van Rillaer M, et al. Development and preliminary evaluation of the validity and reliability of a revised illness perception questionnaire for healthcare professionals. BMC Nurs 2016;15 34 . 
29 Nunnally J, Bernstein I. Psychometric theory. 3rd ed. New York: McGraw-Hill Inc, 1994.

30 Cohen J. Statistical power analysis for the behavioral sciences. 2nd ed, 1988.

31. Worsely C, Whitehead K,Kandler R. et al. Illness perceptions of health care workers in relation to epileptic and psychogenic nonepileptic seizures. Epilepsy Behav 2011;20:668-73.

32. Whitehead K, Reuber M. Illness perceptions of neurologists and psychiatrists in relation to epilepsy and nonepileptic attack disorder. Seizure 2012;21:104-9.

33. Rees G, Lamoureux EL, Xie J, et al. Using rasch analysis to evaluate the validity of the diabetes-specific version of the Illness Perception Questionnaire--Revised. J Health Psychol 2015;20:1340-56.
34 French DP, Weinman J. Current issues and new directions in Psychology and Health: 'Assessing illness perceptions: Beyond the IPQ'. Psychol Heal 2008;23:5-9.

35 Kwakkenbos L, Bluyssen SJ, Vonk MC, et al. Addressing patient health care demands in systemic sclerosis: pre-post assessment of a psychoeducational group programme. Clin Exp Rheumatol 2011;29:S60-5.

36. Poole JL, Mendelson C, Skipper B, et al. Taking charge of systemic sclerosis: a pilot study to assess the effectiveness of an internet selfmanagement program. Arthritis Care Res 2014;66:778-82.

37. Buck U, Poole J, Mendelson C. Factors related to self-efficacy in persons with scleroderma. Musculoskeletal Care 2010;8:197-203. 